functional aetiology. Functional stroke mimics accounted for $8 \%$ of admissions to an acute stroke service in London (Gargalas et al., 2015) and the prevalence and burden of functional stroke is well recognised by clinicians. We present a systematic review and meta-analysis aiming to: 1) estimate the prevalence of stroke mimics and functional stroke mimics across medical settings; and 2) describe the demographic and symptom profiles of functional stroke patients.

Methods Three literature searches took place between 20152018 utilising OvidSP, PubMed, CINAHL and Google Scholar. A total of 13974 abstracts were reviewed and 114 papers met inclusion criteria. Age and sex proportions were compared between stroke, stroke mimic and functional mimic groups. Prevalence rates across settings and moderators of functional mimic rates were calculated using random-effects models.

Results Stroke, stroke mimic and functional mimic definitions varied between studies. Across settings, 25\% of suspected stroke patients were stroke mimics and $15 \%$ of stroke mimics had a functional aetiology. Stroke mimics were younger than stroke patients and more likely to be female. Similarly, functional patients were younger and more often female than medical mimics. 10 papers gave symptom information for functional patients; compared to medical mimics, functional patients were more likely to display weakness/numbness and less likely to present with reduced consciousness, visual symptoms or speech/language symptoms. Meta-analyses show a higher rate of stroke mimics in primary care $(38 \%)$ vs more acute settings $(12 \%)$ but the inverse for functional mimics ( $24 \%$ in stroke units vs only $12 \%$ in primary care). Functional rates were highest in studies that were descriptive, retrospective, from high income countries and in studies where all patients received thrombolysis.

Conclusions Functional diagnoses are an important differential of suspected stroke. Definitions of functional stroke mimics vary widely in stroke literature. Our findings suggest functional stroke patients are most commonly seen in tertiary settings. There are no guidelines on the management of these patients within acute stroke settings. In the context of these findings, a feasibility study is underway investigating the presentation of functional stroke patients and their views on possible interventions and this research may help improve current care pathways.

\section{MEDICATION PRESCRIPTIONS IN 322 FUNCTIONAL MOTOR DISORDER PATIENTS IN A LARGE UK MENTAL HEALTH SERVICE: A CASE CONTROL STUDY}

Nicola O'Connell ${ }^{*}$, Timothy Nicholson, Graham Blackman, Jennifer Tavener, Anthony, S David. Department of Public Health and Primary Care, School of Medicine, Trinity College Dublin

\subsection{6/jnnp-2019-BNPA.36}

Objectives This study aims i) to describe the patterns of prescription medication in functional motor disorder (mFND) treated in a Mental Health Trust, using a control group comprised of a random sample of contemporaneous patients treated in the same trust; and ii) explore the sociodemographic variables and symptoms linked to higher medication usage.

Methods This is a retrospective case-control study using an electronic psychiatric case register in South London and Maudsley NHS Foundation Trust (SLaM). SLaM provides psychiatric inpatient and community services and receives national referrals for functional neurological disorder (FND) patients. Data were obtained from $322 \mathrm{mFND}$ patients and 644 controls between $1^{\text {st } J a n u a r y ~} 2006$ and $31^{\text {st }}$ December 2016 using the 'Clinical Records Interactive Search' (CRIS) database.

Results 247 (76.7\%) mFND patients were prescribed medication, lower than $83.4 \%$ in the control group (OR: 0.59 , 95\% CI: $0.39-0.89, \mathrm{p}<0.02)$. The mean number of prescribed medications in the mFND group was 4.77 (SD: 2.4), higher than 2.98 (SD: 2.7) in the psychiatric control group $(t$ (782) =7.9, $\mathrm{p}=0.001)$. Amongst mFND patients receiving medication, the most common prescriptions were antidepressants (68\% received one or more), anti-epileptics $(33.5 \%$ on one or more), non-opioid painkillers (32.4\%), and opioid analgesics $(31.2 \%)$. Compared to psychiatric controls, mFND patients had a higher likelihood of receiving anti-depressants, medications for cardiovascular disease, statins, antihistamines, antiasthmatics, corticosteroids, anti-epileptics, hormone replacement therapy, proton pump inhibitors, bowel and urinary dysfunction medication, NSAIDs, and muscle relaxants. mFND patients were significantly less likely to receive antipsychotic medication or treatments for substance misuse. An adjusted analysis of mFND patients found co-morbid physical conditions and previous psychiatric admissions were associated with higher numbers of medication prescriptions.

Conclusions mFND patients are prescribed an extensive range of medications for psychiatric and somatic symptoms, most commonly anti-depressants, anti-epileptics and analgesics. The diversity in medications may be partially explained by higher rates of physical co- morbidities but may also reflect 'somatisation' or excessive symptom reporting combined with a lack of therapeutic options for clinicians managing patients with complex functional and 'organic' conditions and chronic pain. Non-essential medication prescribing may reinforce somatic illness beliefs and cause iatrogenic harm, particularly high rates of opioid pain medication, with important implications for clinical management in primary and secondary care.

\section{OUTPATIENT COGNITIVE BEHAVIOURAL THERAPY FOR 'FUNCTIONAL' AND 'ORGANIC' NEUROPSYCHIATRIC DISORDERS: A RETROSPECTIVE CASE CONTROL COMPARISON}

Nicola O'Connell*, Gillian Watson, Clare Grey, Rosa Pastena, Kenneth McKeown, Anthony S David. Department of Public Health and Primary Care, School of Medicine, Trinity College Dublin

\subsection{6/jnnp-2019-BNPA.37}

Objectives There is no gold standard treatment for functional neurological disorder, motor type (mFND). Cognitive behavioural therapy (CBT) is effective in the treatment of certain somatoform disorders. This study aims to evaluate the characteristics and outcomes of mFND patients receiving CBT in a neuropsychiatry outpatient clinic.

Method We utilise a large psychiatric register to assess all mFND patients receiving outpatient CBT in a neuropsychiatric clinic between 2006 and 2011. We assess socio-demographic characteristics, changes in physical and psychological outcomes using standardised rating scales, and rates of CBT uptake and dropout. We compare mFND patients to patients with psychiatric and behavioural manifestations of organic neuropsychiatric diseases treated in the same clinic (ONP patients). 
Results The most common reasons for patients not accepting a CBT referral were referral to the Trust's inpatient neuropsychiatry ward, or non-attendance at assessment or treatment sessions. We identified a total of 98 patients with mFND and 76 ONP patients treated with CBT. $56.1 \%$ of $\mathrm{mFND}$ and $56.6 \%$ of ONP patients attended all sessions offered. There were no socio-demographic characteristics associated with mFND patients who completed all therapy sessions versus those who dropped out early. Compared to controls, mFND patients were more likely to be female $\left(72.4 \% \mathrm{v} .44 .7 \%, \chi^{2}\right.$ : 13.6, 95\% CI: $12.2-41.9, \mathrm{p}=0.001)$, unemployed $(52.6 \%$ versus $35.5 \%, \chi 2: 5,95 \% \mathrm{CI}: 2.2-30.8, \mathrm{p}=0.03)$, to have a carer $(27.6 \%$ versus $14.3 \%, \chi 2: 4.4,95 \%$ CI: 0.9-24.7, $\mathrm{p}=0.04)$ and to have experienced child sexual abuse $(23.8 \% \mathrm{v}$ $\left.8.2 \%, \chi^{2}: 7.3, p=0.01\right)$. The most common mFND symptom was weakness $(26.9 \%)$, most frequently in the leg or entire body. Both mFND and ONP patients showed significant improvements in measures of psychological functioning (measured with the CORE-OM, HoNOS-ABI, and PHQ-9), with physical symptoms improving in $49.4 \%$ of mFND patients. A logistic regression showed acceptance of psychological formulations prior to CBT $(\mathrm{p}<0.02)$ was associated with the improvement of physical symptoms in mFND patients.

Conclusions mFND patients treated in a specialist CBT clinic show similar improvements in physical and psychological functioning to ONP patients with positive effects on motor symptoms, distress, depression, general health and social functioning. With little evidence on the effectiveness of CBT in treating motor variants of FND, this study could help inform the design of a pilot RCT.

\section{8 \\ ARE THERE ANY FACTORS THAT PREDICT THE DIAGNOSIS OF EPILEPSY OR PSYCHOGENIC NON-EPILEPTIC SEIZURES (PNES) IN PATIENTS ADMITTED TO A SPECIALIST EPILEPSY UNIT?} ${ }^{1} \mathrm{~S}$ Gibson*, ${ }^{2} \mathrm{~S}$ Leighton, ${ }^{1,2} \mathrm{M}$ Oto. ${ }^{1} \mathrm{NHS}$ Greater Glasgow and Clyde; ${ }^{2}$ William Quarrier
Scottish Epilepsy Centre

\subsection{6/jnnp-2019-BNPA.38}

Introduction Differentiating between epilepsy and psychogenic non-epileptic seizures (PNES) can be difficult. Although clearly not a substitute for taking a careful history, certain patient characteristics may assist the clinicians towards diagnosis. The population of patients referred to an epilepsy specialist centre represent a complex and distinct group of patients and it is not clear which factors, if any, could point towards a diagnosis of epilepsy or PNES.

Aims/Objectives To investigate if the diagnosis of epilepsy or PNES is predicted by baseline demographic and clinical variables, including Hospital Anxiety and Depression (HADS) scores and medication prescription, in patients admitted to a specialist adult epilepsy centre.

Methods We conducted an observational retrospective cohort of consecutive patients admitted to the William Quarrier Scottish Epilepsy Centre (WQSEC) over a period of one year (01/ 09/16-01/09/17). Chosen predictor variables at baseline included: sex, age, employment education or training (EET), Scottish Index of Multiple Deprivation 2016 (SIMD 2016) rank status, attack frequency, length of index admission, number of anti-epileptic agents prescribed, prescription of benzodiazepines, of analgesia, or of psychotropic medications, and
HADS scores. Outcome measures were diagnosis of epilepsy or PNES, from diagnosis made by expert clinicians on discharge from index admission. Because of the presence of dual diagnosis, two multivariable binary logistic regression models were built - one for the epilepsy and one for the PNES diagnosis outcomes.

Results 50/73 (69\%) of patients admitted were diagnosed with epilepsy and 39/73 (53\%) with PNES. These respective groups include 16/73 (22\%) who had a dual diagnosis of both epilepsy and PNES. The model to predict epilepsy showed that significant individual predictor variables included number of antiepileptic agents prescribed (Odds Ratio (OR) $=3.59 \quad(95 \%$ CI: $1.37,9.42), p=0.010)$, prescription of psychotropic medications $(\mathrm{OR}=0.19(95 \% \mathrm{CI}: 0.04,0.91), \mathrm{p}=0.038)$, and length of index admission (OR=0.89 (95\%CI: $0.81,0.98)$, $\mathrm{p}=0.018$ ). The model to predict presence of PNES revealed only one significant individual predictor variable, which was EET status (OR 0.13 (95\%CI: 0.02, 0.86), p=0.035).

Conclusions Baseline clinical and demographic factors may be of some utility to the clinician in anticipating a diagnosis of epilepsy or of PNES.

\section{THE IMMUNOMODULATORY EFFECT OF CLOZAPINE IN PATIENTS WITH TREATMENT RESISTANT SCHIZOPHRENIA: A RETROSPECTIVE COHORT STUDY}

${ }^{1}$ Lisshammar J, ${ }^{1}$ Blackman G, ${ }^{1}$ Carter B, 'Zafar R, ' Stewart R, ${ }^{1}$ Pritchard M, ${ }^{1}$ Pollak T, ${ }^{1}$ Rogers J, ${ }^{1}$ Cullen A, ${ }^{1}$ McGuire P, ${ }^{2}$ David A, ${ }^{1}$ MacCabe J. ${ }^{1}$ Institute of Psychiatry, Psychology and Neuroscience, King's College London, Dr Crespigny Park, London, UK.; ' Institute of Mental Health, University College London, London, UK

\subsection{6/jnnp-2019-BNPA.39}

Objectives The pathoetiology of Schizophrenia remains elusive, however, a growing body of literature suggests immune dysfunction may contribute. Clozapine, an atypical antipsychotic, has superior efficacy in treatment-resistant Schizophrenia compared to other antipsychotics - however underlying mechanisms remain unknown. Clozapine has recognised immunomodulatory effects, responsible for potentially fatal haematological side-effects - such as agranulocytosis. Whether Clozapine's immunomodulatory properties contribute toward its unique efficacy in treatment-resistant schizophrenia has not been systematically explored.

Methods A retrospective cohort study design was employed to examine the relationship between white cell, neutrophil, and platelet temporal trajectories and Clozapine response in treatment-resistant schizophrenia. Eligible patients were initiated on Clozapine for the first time and continued treatment for at least twelve weeks between 2007 and 2014 within the South London and Maudsley NHS Foundation Trust, and underwent weekly haematological monitoring. Retrospective clinical ratings were performed at baseline and three months following initiation, based upon patients' electronic clinical notes accessed through the Maudsley BRC Clinical Records Interactive Search system. Treatment response was defined as 'much' or 'very much' improved on the Clinical Global Impression Improvement subscale. Serial cell counts were extracted from a Clozapine haematological monitoring database.

Results Of 188 included patients, 114 (61\%) responded to treatment. Response did not significantly vary by ethnicity or age. Mean interval between haematological assessments was 6.9 (SD 2.0) days. General linear models revealed a significant increase from baseline for all cell lines in the second 